\title{
Microscopic Theory of Hydrogen in Silicon Devices
}

\author{
Chris G. Van de Walle, Member, IEEE, and Blair R. Tuttle
}

Invited Paper

\begin{abstract}
Incorporation of hydrogen has a strong effect on the characteristics of silicon devices. A fundamental understanding of the microscopic mechanisms is required in order to monitor and control the behavior of hydrogen. First-principles calculations have been instrumental in providing such understanding. We first outline the basic principles that govern the interaction between hydrogen and silicon, followed by an overview of recent first-principles results for hydrogen interactions with silicon. We show that $\mathrm{H}_{2}$ molecules are far less inert than previously assumed. We then discuss results for motion of hydrogen through the material, as relating to diffusion and defect formation. We also discuss the enhanced stability of $\mathrm{Si}-\mathrm{D}$ compared to $\mathrm{Si}-\mathrm{H}$ bonds, which may provide a means of suppressing defect generation. We present a microscopic mechanism for hydrogen-hydrogen exchange, and examine the metastable $\equiv \mathrm{SiH}_{2}$ complex formed during the exchange process. Throughout, we highlight issues relevant for hydrogen in amorphous silicon (used in solar cells, sensors and displays) and in $\mathrm{Si}_{-} \mathrm{SiO}_{2}$ structures (used in integrated circuits). The broader impact of first-principles calculations on computational electronics will also be discussed.
\end{abstract}

Index Terms-Amorphous semiconductors, hydrogen, semiconductor defects, semiconductor impurities, semiconductor/insulator interfaces, silicon.

\section{INTRODUCTION}

$\mathbf{H}$ YDROGEN plays an important role in many technologically relevant processes in silicon [1]-[8]. Introduction of hydrogen can result in the passivation of shallow acceptor and donor states, as well as of electrically active deep levels [1]. The latter are commonly associated with silicon dangling bonds and are found at surfaces, grain boundaries, interfaces, and in bulk silicon. Incorporation of hydrogen during the growth of amorphous silicon ( $a$-Si) films is essential for producing devices such as solar cells [2]. Also, device-quality silicon-based metal-oxide-semiconductor field effect transistors (MOSFETs) are annealed in a hydrogen-rich environment in order to passivate defects at the $\mathrm{Si}^{-} \mathrm{SiO}_{2}$ interface [7], [8]. The use of hydrogen to passivate defects in these devices sets the stage for the

Manuscript received February 11, 2000. The work of B. R. Tuttle was supported by Xerox Foundation, National Science Foundation (through DesCartES), and the Office of Naval Research (MURI Grant N00014-98-I-0604). The work of C. Van de Walle was supported by the Fritz-Haber-Institut and Paul-Drude-Institut and the Alexander von Humboldt Foundation through a U.S. Senior Scientist Award. The review of this paper was arranged by Editor R. W. Dutton.

C. G. Van de Walle is with Xerox Palo Alto Research Center, Palo Alto, CA 94304 USA (e-mail: vandewalle@parc.xerox.com).

B. R. Tuttle is with the Beckman Institute, University of Illinois, Urbana, IL 61801 USA

Publisher Irem Identifier S 0018-9383(00)07776-5. subsequent creation of defects during device operation. For instance, hydrogen is known to play an important role during hotelectron degradation in MOSFETs [9], [10], as well as during light-induced degradation in $a-\mathrm{Si}: \mathrm{H}$ solar cells [3], [11]. Use of deuterium has been shown to lead to a suppression of degradation [5], [6], [9]. A fundamental understanding of the role of hydrogen in these devices is very much desired.

The interaction between hydrogen and the semiconductor takes many forms. Isolated interstitial hydrogen already displays a strong tendency to disrupt the normal bonding in the material: in the positive and neutral charge states hydrogen inserts in a bond-center (BC) site in silicon. Hydrogen also interacts strongly with shallow as well as deep impurities, as well as with other hydrogen atoms, resulting in $\mathrm{H}_{2}$ molecule formation for example. Last but not least, hydrogen interacts with intrinsic defects, the passivation of dangling bonds being the best known example.

It is highly desirable to develop theoretical and computational models that capture this multifaceted behavior of hydrogen within silicon devices. Purely phenomenological approaches may be appropriate for isolated aspects of hydrogen's interaction with the semiconductor, but to describe the full complexity involved in bonding, diffusion, passivation, defect creation, etc., a first-principles approach at the atomic level is required. Such first-principles calculations not only answer quantitative questions, but more importantly provide a theoretical framework in which the underlying fundamental mechanisms can be examined and categorized. Once these mechanisms are known, higher-level models can be built upon them. For instance, the first-principles calculations elucidate the importance of taking the proper charge state of interstitial hydrogen into account. This information can then be included when developing a model for hydrogen diffusion as part of a process-simulation package. As another example, one may wish to model device degradation due to defect creation at the $\mathrm{Si} / \mathrm{SiO}_{2}$ interface, and the enhanced stability offered by deuterium passivation. In order to construct a reliable model, the basic mechanisms of defect creation due to breaking of $\mathrm{Si}-\mathrm{H}$ or $\mathrm{Si}-\mathrm{D}$ bonds must be understood. Again, this fundamental information can be produced by first-principles calculations.

In this paper, we will review recent work that illustrates the power of this approach. Specifically, we will focus on the role of hydrogen in $a-\mathrm{Si}$ and at the $\mathrm{Si}-\mathrm{SiO}_{2}$ interface. The computational results have all been obtained using a state-of-the-art first-principles approach based on density-functional theory, $a b$ initio pseudopotentials, and a supercell geometry. 
After a brief review of the methods (Section II), we will describe some basic principles that govern the behavior of hydrogen in silicon (Section III). Section IV will focus on hydrogen molecules: we will review experimental observations of interstitial $\mathrm{H}_{2}$ molecules in crystalline and amorphous semiconductors, and describe the theoretical framework for understanding the physics of incorporation of a strongly bound molecule in a semiconducting environment. Section $V$ will deal with hydrogen mobility and desorption, as occurs in hydrogen diffusion, hot electron degradation and light-induced defect generation. We will first discuss the mechanism for thermal desorption, and then turn to the dissociation mechanism of $\mathrm{Si}-\mathrm{H}$ bonds and the connection to vibrational properties of the system. We will show how these insights into the microscopic mechanisms immediately explain the enhanced stability of Si-D bonds. We will also discuss an exchange process between trapped and interstitial hydrogen that plays a significant role in diffusion processes. We have determined a low-energy pathway for exchange which involves an intermediate, metastable $\equiv \mathrm{SiH}_{2}$ complex with both hydrogen atoms strongly bound to the silicon atom. Again, the emphasis of our discussion will be on the role of hydrogen in $a-\mathrm{Si}$ and at the $\mathrm{Si}-\mathrm{SiO}_{2}$ interface.

Finally, in Section VI, we provide a brief summary and also an outlook on the future role of first-principles calculations, both for studying hydrogen and for broader applications.

\section{METHODS}

We have used a state-of-the-art first-principles approach based on density-functional theory in the local-density approximation [12]. Wave functions and potentials are expanded in a plane-wave basis set, and we employ a supercell geometry, with $a b$ initio pseudopotentials for the semiconductor host atoms [13], [14]. Relaxation of host atoms is always included, and 32-atom supercells are typically used. This approach has produced reliable results for bulk properties of many materials, as well as properties of surfaces, interfaces, impurities, and defects. More details about the application of the method to the study of hydrogen can be found in [15]-[17]. We estimate the uncertainty on the energies quoted here to be $\pm 0.1 \mathrm{eV}$.

\section{BASIC PRINCIPLES GOVERNING HydROGEN INTERACTIONS}

\section{A. Isolated Interstitial Hydrogen}

The atomic and electronic structure of isolated interstitial hydrogen depends strongly on its charge state, which can be positive, neutral, or negative. In the positive charge state the impurity is essentially a proton, which is electrostatically attracted to regions of high charge density. In silicon, the charge density is highest at the bond center. In order to accommodate the proton at this location, the host atoms have to move outwards, which costs energy. In spite of this cost, the three-center bond formed between $\mathrm{H}$ and the host atoms is sufficiently strong to stabilize this configuration [15]. In the negative charge state, the $1 s$ shell is filled, leading to a diminished tendency for $\mathrm{H}$ to interact with the host atoms. The negative charge also compels the $\mathrm{H}^{-}$ to maximize its distance to the host atoms; it therefore prefers to be located at a tetrahedral interstitial $\left(T_{d}\right)$ site.
Hydrogen assumes these different charge states through exchange of electrons with the Fermi level. To form $\mathrm{H}^{+}$, an electron has to be donated to the Fermi level, a process that is most favorable when the Fermi energy is low, i.e., in p-type material. $\mathrm{H}^{-}$, on the other hand, is most favorable in n-type material. The transition levels between these various charge states have been determined theoretically as well as experimentally in $\mathrm{Si}$ [18]. The donor level occurs near the conduction band; the acceptor level is located near midgap. The ordering of these levels indicates that hydrogen is a so-called "negative- $U$ " center; this implies that the neutral charge state is never stable in equilibrium. As discussed in [19] the negative- $U$ character is likely a universal feature of hydrogen in any semiconductor.

For $\mathrm{H}^{0}$ at the $\mathrm{BC}$ site, the energy is $1.05 \mathrm{eV}$ below the energy of $\mathrm{H}^{0}$ in free space. This value agrees with an analysis of solubility data in $c-\mathrm{Si}$ [20]. Interstitial hydrogen is known to diffuse very quickly through silicon. Hydrogen diffusion experiments in $c$-Si [21] indicate that the diffusion barrier is about $0.5 \mathrm{eV}$.

\section{B. Hydrogen Interactions with Shallow Impurities}

Hydrogen passivation of shallow impurities can be readily understood on the basis of the properties of isolated interstitial hydrogen. In p-type material, $\mathrm{H}^{+}$is the preferred charge state. $\mathrm{H}^{+}$ will diffuse toward electricallly active, negatively charged acceptors, to which it is Coulombically attracted. A complex is formed, which is electrically inactive, and in which the hydrogen is located in its preferred position in p-type material (i.e., the BC position). Similar arguments apply to $\mathrm{H}$ in n-type semiconductors: $\mathrm{H}^{-}$will seek out positively charged donors, and assume an antibonding position (close to the $T_{d}$ site) in the complex.

Passivation of shallow impurities by hydrogen is, in general, very undesirable. Such passivation eliminates the intended electrical activity of the dopants and therefore strongly affects device behavior. Since hydrogen is omnipresent during growth and processing of semiconductor devices, its effects on shallow impurities need to be carefully scrutinized. In silicon, hydrogen passivation of acceptors as well as donors can be readily eliminated by annealing the material at modest temperatures (above $150^{\circ} \mathrm{C}$ ), leading to dissocation of the dopant-hydrogen complexes and neutralization of the hydrogen.

\section{Hydrogen-Hydrogen Interactions; $\mathrm{H}_{2}$ Molecules}

$\mathrm{H}_{2}$ molecules can easily form in most semiconductors; the binding energy is somewhat smaller than for $\mathrm{H}_{2}$ in vacuum, but still large enough to make interstitial $\mathrm{H}_{2}$ one of the more favorable configurations hydrogen can assume in the lattice. $\mathrm{H}_{2}$ molecules have commonly been assumed to be present in many semiconductors, but experimental observations have only recently been reported. To aid in the interpretation of the experimental results, and to further our understanding of the physics of $\mathrm{H}_{2}$ incorporation, we have performed comprehensive first-principles computational studies of interstitial $\mathrm{H}_{2}$. The results are discussed in Section IV.

\section{Formation of Strong Bonds with Host Atoms}

Strong bonds between hydrogen and host atoms can be formed when some disruption in the perfect crystal is present; 
for instance, at a surface, at an interface, in polycrystalline or amorphous material, or near a point defect in the bulk. The formation of such strong bonds between hydrogen and host atoms is often implicitly considered to be due to the passivation of dangling bonds. It is indeed often assumed that intrinsic deep levels are all due to dangling bonds. However, one should not only focus on undercoordination defects (dangling bonds), but also consider the occurrence of overcoordination defects. Indeed, in crystalline $\mathrm{Si}$ it has been accepted for some time that vacancies are not the only type of intrinsic defect to play a role. Self-interstitials have formation energies comparable to those of vacancies [22]. In amorphous silicon, too, overcoordination defects may occur, in addition to undercoordinated atoms, as pointed out by Pantelides [23]. In crystalline silicon, the self-interstitials are known to be involved in self-diffusion, impurity diffusion, surface reconstructions, planar interstitial defect formation, and dislocation nucleation (see [24] for references).

First-principles calculations for complexes consisting of one or two $\mathrm{H}$ atoms and a Si self-interstitial were reported in [24], addressing atomic structure, electronic structure, and vibrational frequencies. It was found that hydrogen interacts strongly with self-interstitials; while the calculated binding energy is smaller than for $\mathrm{H}$ interacting with a vacancy, it is large enough for the complexes to be stable at room temperature. The electronic structure of the complex between a self-interstitial and one $\mathrm{H}$ atom indicates that it is amphoteric in nature. The complex with two hydrogens has no levels in the band gap, consistent with all the bonds being satisfied.

The more commonly considered case is that of hydrogen interacting with a dangling bond. Until recently, little information was available on the energetics of the $\mathrm{Si}-\mathrm{H}$ bond in bulk (crystalline or amorphous) $\mathrm{Si}$. It had mostly been assumed that the bond strength would be similar to that in a silane $\left(\mathrm{SiH}_{4}\right)$ molecule; this approach ignores effects of the crystalline environment and possible distortions of the bonding configuration. As a first step, a study was performed for Si-H bonds in a crystalline environment [25]. It was found that the energy cost for removing the $\mathrm{H}$ atom from a Si-H bond, leaving a dangling bond behind, is $3.55 \mathrm{eV}$. Another way to define an energy for the $\mathrm{Si}-\mathrm{H}$ bond is to assume that one starts from crystalline silicon and a hydrogen atom in free space, and that the energy to create a dangling bond needs to be taken into account; this defines a formation energy. For an "ideal" Si-H bond (meaning it is located at a dangling bond which is isolated from other dangling bonds, with no $\mathrm{H}-\mathrm{H}$ repulsion) the formation energy was found to be $-2.17 \mathrm{eV}$. The difference with the energy at $-3.55 \mathrm{eV}$ mentioned above is that one energy includes the formation of a dangling bond while the other does not; the difference is $-2.17-(-3.55)=1.38 \mathrm{eV}$, a value which constitutes an estimate for the formation energy of a dangling bond in $c$-Si.

It is to be expected that the energy of an $\mathrm{Si}-\mathrm{H}$ bond in $a$ - $\mathrm{Si}: \mathrm{H}$ is different from $c$-Si. Indeed, explicit simulations for $\mathrm{Si}-\mathrm{H}$ bonds in amorphous networks [17] show that the Si-H bond energies can be significantly higher than the value for $\mathrm{H}$ at an ideal, isolated dangling bond, due to hydrogen clustering and $\mathrm{H}-\mathrm{H}$ repulsion effects.

\section{HYDROGEN MOLECULES}

It has been known for some time that $\mathrm{H}_{2}$ molecules are one of the most stable forms of hydrogen in semiconductors. This knowledge was based on computational studies (see, e.g., [15], [16], [26]) as well as on interpretation of experimental data. Direct observation of $\mathrm{H}_{2}$ molecules proved very difficult, however, because of sensitivity problems in techniques such as NMR (nuclear magnetic resonance) and vibrational spectroscopy.

A thorough understanding of the incorporation of $\mathrm{H}_{2}$ in the lattice is essential for the many technologically important processes that involve hydrogen: passivation of defects at the $\mathrm{Si}-\mathrm{SiO}_{2}$ interface; the "smartcut" process for producing silicon-on-insulator structures [27]; passivation and generation of defects in amorphous silicon; etc. In amorphous silicon, it has long been known that much more hydrogen is incorporated than is strictly needed for defect passivation. Work by Norberg et al. [28] suggests that a large fraction of this hydrogen could be in the form of interstitial molecules. For many of these processes, it is essential to understand how $\mathrm{H}_{2}$ interacts with existing defects or contributes to the formation of new defects; aspects of such interactions are also addressed in Section V.

\section{A. First-Principles Calculations of Interstitial Hydrogen Molecules}

We have performed first-principles computational studies of interstitial $\mathrm{H}_{2}$ in $\mathrm{Si}$ as well as in a number of other semiconductors [29]. In addition, we have examined $\mathrm{H}_{2}$ in crystalline silicon dioxide $\left(c-\mathrm{SiO}_{2}\right)$ in the low-energy $\alpha$-cristobalite phase [30]. These investigations show that, compared to $\mathrm{H}_{2}$ gas, incorporation of $\mathrm{H}_{2}$ into an interstitial position in semiconductors results in a lowering of the binding energy, an increase in the bond length, and a lowering of the vibrational frequency. These effects can be attributed to the immersion of the molecule in a low-density electron gas near the interstitial site. Indeed, the decrease in binding energy and corresponding lowering of the vibrational frequency correlate with the charge density near the interstitial site. The open $\mathrm{SiO}_{2}$ network in $\alpha$-cristobalite allows the charge density at interstitial sites to become very low; little change is therefore observed between the properties of $\mathrm{H}_{2}$ in $\mathrm{SiO}_{2}$ versus in the gas phase. The calculated difference between the energy of interstitial $\mathrm{H}_{2}$ in $c-\mathrm{SiO}_{2}(\alpha$-cristobalite) and the energy of $\mathrm{H}_{2}$ in vacuum is less than $0.1 \mathrm{eV}$ per molecule. For $c-\mathrm{Si}$, the energy difference is $0.8 \mathrm{eV}$ per molecule. Our calculated lowering of the vibrational stretch frequency for $\mathrm{H}_{2}$ in $\mathrm{Si}$ agrees well with the experimental value [31].

\section{B. Diffusion of $\mathrm{H}_{2}$}

We have performed calculations for diffusion of interstitial $\mathrm{H}_{2}$ in $c-\mathrm{Si}$. We found that the saddle point occurs at the hexagonal interstitial site, with a migration barrier of $0.95 \mathrm{eV}$. An alternative diffusion mechanism for $\mathrm{H}_{2}$ diffusion consists of dissociation of the molecule, followed by atomic diffusion. Results obtained in [16] indicate that dissociation of $\mathrm{H}_{2}$ into two neutral interstitial hydrogen atoms costs $1.74 \mathrm{eV}$. Dissociation into an $\mathrm{H}^{+}-\mathrm{H}^{-}$pair would cost $1.34 \mathrm{eV}$. It seems therefore likely that $\mathrm{H}_{2}$ would diffuse in the molecular form. The calculated barrier agrees with the observed diffusion of interstitial $\mathrm{H}_{2}$ reported in [32]. 


\section{Hydrogen Molecules in Amorphous Silicon and at the $\mathrm{Si}-\mathrm{SiO}_{2}$ Interface}

Many of our results for $\mathrm{H}_{2}$ in crystalline silicon are likely to apply to amorphous silicon as well. The presence of $\mathrm{H}_{2}$ molecules in $a$-Si had been considered previously, but mainly in the context of molecular hydrogen trapped in voids or microbubbles [33], [34]. Device-quality hydrogenated amorphous silicon typically contains up to $15 \%$ hydrogen-a concentration that is much larger than the amount of hydrogen needed to passivate defects. The configuration in which this massive amount of hydrogen is incorporated has been debated for a long time. Norberg and coworkers [28] have recently performed deuteron and proton nuclear magnetic resonance (NMR) measurements on high-quality plasma-enhanced CVD $a$-Si films, showing that up to $40 \%$ of the hydrogen in these samples is not involved in Si-H bonds. On the basis of their measurements they conclude that nearly all of this nonbonded hydrogen is present as isolated $\mathrm{H}_{2}$ molecules, located in centers of atomic dimensions, perhaps in the analogue of $T_{d}$-sites in crystalline silicon. This nonbonded hydrogen also appears to be in the vicinity of light-induced defects, suggesting the molecular hydrogen may play a role in Staebler-Wronski degradation [3]. We will return to this issue in Section V.

Much of our current understanding of the physical chemistry of hydrogen at the $\mathrm{Si}-\mathrm{SiO}_{2}$ interface is based on the experiments and analysis of Brower et al. [7], [8]. In their analysis, it was assumed that atomic $\mathrm{H}$ and $\mathrm{H}_{2}$ would only reside on the $\mathrm{SiO}_{2}$ side of the interface. This led to an estimate of the $\mathrm{H}_{2}$ binding energy in $\mathrm{SiO}_{2}$ being more than $0.4 \mathrm{eV}$ lower than that of $\mathrm{H}_{2}$ gas. In contrast, our calculations indicate the difference is less than $0.1 \mathrm{eV}$ per molecule. This apparent contradiction can be resolved by noting that atomic $\mathrm{H}$ and $\mathrm{H}_{2}$ can also reside and diffuse on the $c$-Si side of the interface. In addition, the amorphous nature of the $\mathrm{SiO}_{2}$ network leads to a distribution of sizes of interstitial cages, and hence a distribution of energies for interstitial $\mathrm{H}_{2}$. Some of these energies may be higher than that calculated for $\alpha$-cristobalite. A quantitative investigation of these issues requires full first-principles calculations of $\mathrm{H}$ in various charge states in $\mathrm{SiO}_{2}$, as well as in $\mathrm{Si} \mathrm{[10].} \mathrm{Several}$ groups have recently started explicit first-principles investigations of select configurations of $\mathrm{H}$ in $\mathrm{SiO}_{2}$ [10], [35]-[37]. Some other aspects of hydrogen at $\mathrm{Si} / \mathrm{SiO}_{2}$ interfaces will be discussed below.

\section{HYDROGEN MOBILITY AND DESORPTION}

Interstitial hydrogen can diffuse through crystalline silicon with an activation energy of about $0.5 \mathrm{eV}$ [21]. Hydrogen interacts strongly with other impurities as well as with defects in the crystal. The strongest of these interactions is with silicon dangling bonds, where $\mathrm{Si}-\mathrm{H}$ bonds are formed with bond strengths up to $3.6 \mathrm{eV}$ [16], [17], similar to those in silane. Silicon dangling bonds thus form deep traps for hydrogen. As noted in the introduction, hydrogen is used to passivate silicon dangling bond defects in both $a-\mathrm{Si}: \mathrm{H}$ solar cells and MOSFETs. This sets the stage for device degradation when hydrogen atoms dissociate and diffuse away from silicon dangling bonds.

\section{A. Si-H Dissociation by Thermal Excitation}

During thermal annealing in vacuum, hydrogen can be removed from bulk $a-\mathrm{Si}: \mathrm{H}$ and the $\mathrm{Si}-\mathrm{SiO}_{2}$ interface at temperatures above 200 and $500{ }^{\circ} \mathrm{C}$, respectively. Brower [7] has observed the kinetics of silicon dangling bond creation during the thermal desorption of hydrogen from the $\mathrm{Si}(111)-\mathrm{SiO}_{2}$ interface. They determined that the rate-limiting step is activated with a barrier of $2.6 \mathrm{eV}$. The energy to remove a hydrogen atom from an $\mathrm{Si}-\mathrm{H}$ bond and place it in free space or in an open interstitial of $c-\mathrm{SiO}_{2}$ [30] is $3.6 \mathrm{eV}$. However, only $2.5 \mathrm{eV}$ is needed to place the hydrogen in an interstitial site in bulk $c-\mathrm{Si}$. Even less energy may be required, because both the $\mathrm{H}$ interstitial and the silicon dangling bond can become charged, thus lowering the energy of the final state. Once in the interstitial site, the $\mathrm{H}$ atom is mobile and has a migration barrier of less than $0.5 \mathrm{eV}$. Interstitial hydrogen will subsequently lower its energy, e.g., by binding to other defects or by forming an $\mathrm{H}_{2}$ molecule. At surfaces or interfaces with open materials such as $\mathrm{SiO}_{2}, \mathrm{H}_{2}$ can easily escape from the material. Overall, the $\mathrm{Si}-\mathrm{H}$ dissociation energy barrier is expected to be less than $3.0 \mathrm{eV}$ [38].

Analysis of diffusion data for $a-\mathrm{Si}: \mathrm{H}$ produces an activation barrier of $1.5 \mathrm{eV}$ [39], i.e., much lower than the barrier for $\mathrm{H}$ desorption from the $\mathrm{Si}-\mathrm{SiO}_{2}$ interface. This indicates the diffusion process in $a$-Si cannot involve the same type of bond breaking that would occur at the surface or at an $\mathrm{Si}-\mathrm{SiO}_{2}$ interface. At the $\mathrm{Si}-\mathrm{SiO}_{2}$ interface, $\mathrm{Si}-\mathrm{H}$ are separated by about $100 \AA$ whereas in $a-\mathrm{Si}: \mathrm{H}$ over half the $\mathrm{Si}-\mathrm{H}$ bonds are clustered within 2-3 $\AA$ from each other [17]. The lower barrier for $\mathrm{H}$ desorption in $a-\mathrm{Si}: \mathrm{H}$ may therefore be attributed to this clustering. Specifically, these clustered $\mathrm{Si}-\mathrm{H}$ bonds dissociate in pairs with a weak $\mathrm{Si}-\mathrm{Si}$ bond forming after the two $\mathrm{H}$ atoms diffuse away [30], [40]. Assuming atomic $\mathrm{H}$ results from the $\mathrm{Si}-\mathrm{H}$ bond breaking process, we calculate the energetics of this process to be in good agreement with the observed activation energy of $\sim 1.5 \mathrm{eV}$ [30]. It is also possible that $\mathrm{H}_{2}$ forms during the dissociation of clustered $\mathrm{Si}-\mathrm{H}$ bonds. The activation energy would then correspond to the energy cost to produce two atomic $\mathrm{H}$, minus the energy gained by forming $\mathrm{H}_{2}$, plus the diffusion barrier for $\mathrm{H}_{2}$.

\section{B. Si-H Dissociation by Electronic Excitation; H/D Isotope Effect}

Experiments have shown that $\mathrm{Si}-\mathrm{D}$ bonds behave very differently from $\mathrm{Si}-\mathrm{H}$ under electronic excitation: $\mathrm{Si}-\mathrm{D}$ bonds were found to be orders of magnitude harder to break. This giant isotope effect was first observed for $\mathrm{Si}-\mathrm{H}$ bonds on Si surfaces [5], [6], and quickly applied to passivation of defects at the $\mathrm{Si}-\mathrm{SiO}_{2}$ interface in MOSFETs [9]. Since hydrogenated amorphous Si suffers from carrier- and light-induced degradation, it should be expected that the observed enhanced stability of Si-D as compared to $\mathrm{Si}-\mathrm{H}$ would also apply to $\mathrm{Si}-\mathrm{D}$ bonds in $a$-Si. Experimental observations of the enhanced stability of deuterated 
$a-\mathrm{Si}$ under light exposure were recently reported by Wei et al. [41] and by Sugiyama et al. [42]. Replacing hydrogen with deuterium has also been reported to greatly reduce the PL degradation of porous silicon [43].

These observations are surprising, because $H$ and $D$ are entirely equivalent from an electronic point of view: indeed, the static electronic structure of the $\mathrm{Si}-\mathrm{H}$ and $\mathrm{Si}-\mathrm{D}$ bonds is identical. The difference must therefore be attributed to dynamics. We have proposed a mechanism which provides a natural explanation for the difference in dissocation rates [44]. The dissociation of $\mathrm{Si}-\mathrm{H}$ bonds has been proposed to proceed via multiple-vibrational excitation by tunneling electrons (at least in the low-voltage regime) [45]. The extent to which vibrational energy can be stored in the bond depends on the lifetime, i.e., on the rate at which energy is lost by coupling to phonons. Because the lifetime of $\mathrm{H}$ on $\mathrm{Si}$ is long [46], [47], efficient vibrational excitation is expected. The question then is: why would $\mathrm{Si}-\mathrm{D}$ behave qualitatively differently?

It turns out that the path followed by the hydrogen (or deuterium) atom during the breaking of a $\mathrm{Si}-\mathrm{H}$ ( $\mathrm{Si}-\mathrm{D})$ bond plays a crucial role in the dissociation mechanism. It was often implicitly assumed that dissociation would proceed by moving the $\mathrm{H}$ atom away from the $\mathrm{Si}$ along the direction of the $\mathrm{Si}-\mathrm{H}$ bond away from the Si atom; however, this is unlikely to be the most favorable path, for two reasons: a) the initial rise in energy in that direction is high, as indicated by the high vibrational frequency (around $2100 \mathrm{~cm}^{-1}$ ) for the $\mathrm{Si}-\mathrm{H}$ stretch mode; b) this path eventually leads to a position of the $\mathrm{H}$ atom in the interstitial channel, which is not the lowest energy site for $\mathrm{H}$ in the neutral or positive charge state (in $c$-Si). Both of these arguments actually favor a different path in which the $\mathrm{H}$ atom stays at approximately constant distance from the $\mathrm{Si}$ atom to which it is bound: 1) the barrier in that direction is much lower, as indicated by the vibrational frequency (around $650 \mathrm{~cm}^{-1}$ ) for the $\mathrm{Si}-\mathrm{H}$ bending mode; 2 ) this path leads to $\mathrm{H}$ positions closer to the $\mathrm{Si}$ atom, which are more favorable for $\mathrm{H}^{0}$ and $\mathrm{H}^{+}$in $c$-Si. We recently presented a detailed examination of these dissociation paths [38].

The vibrational lifetime is thus mostly controlled by the $\mathrm{Si}-\mathrm{H}$ bending modes. The vibrational frequency of the bending mode for $\mathrm{Si}-\mathrm{H}$ is around $650 \mathrm{~cm}^{-1}$, and the estimated frequency for $\mathrm{Si}-\mathrm{D}$ is around $460 \mathrm{~cm}^{-1}$. The latter frequency turns out to be very close to the frequency of bulk TO phonon states at the $X$ point $\left(463 \mathrm{~cm}^{-1}\right)$ [48]. We therefore expect the coupling of the $\mathrm{Si}-\mathrm{D}$ bending mode to the Si bulk phonons to result in an efficient channel for deexcitation. While it is quite possible to reach a highly excited vibrational state in the case of $\mathrm{Si}-\mathrm{H}$, this will be more difficult for $\mathrm{Si}-\mathrm{D}$. These qualititative differences between $H$ and $D$ have recently been confirmed in tight-binding molecular dynamics studies by Biswas et al. [49]. Deuterium should therefore be much more resistant to STM-induced desorption and hot-electron induced dissociation, due to the relaxation of energy through the bending mode.

Displacements along the "bond-bending path" also cause energy levels to be introduced into the band gap (near the valence band and near the conduction band), enabling the complex to capture carriers; after changing charge state there is virtually no barrier to further dissociation. The barrier for dissociation can therefore be significantly reduced when carriers are present, such as in a scanning tunneling microscope (STM) desorption experiment; at an $\mathrm{Si} / \mathrm{SiO}_{2}$ interface, during device operation of the MOSFETs; or in $a-\mathrm{Si}$, in the form of injected or light-induced carriers.

\section{Exchange of Deeply Trapped and Interstitial Hydrogen}

Hydrogen exchange between deeply trapped and mobile states plays an important role in the diffusion process [50]. If such exchange takes place by first dissociating a $\mathrm{Si}-\mathrm{H}$ bond and subsequently placing another $\mathrm{H}$ at the dangling bond, the activation energy would be prohibitively high. Experimentally, however, the exchange is known to proceed very efficiently, based on observations of deuterium replacement of hydrogen in $a-\mathrm{Si}: \mathrm{H}[51]-[53]$ and at the $\mathrm{Si}-\mathrm{SiO}_{2}$ interface of MOSFETs [9]. Unraveling the microscopic mechanisms by which a neutral interstitial hydrogen can exchange with a deeply trapped hydrogen was a challenge we tackled with first-principles calculations [54].

Our main result is that $\mathrm{H}-\mathrm{H}$ exchange can proceed with an energy barrier of less than $0.2 \mathrm{eV}$. The first part in the process consists of an interstitial $\mathrm{H}$ atom approaching the $\mathrm{Si}-\mathrm{H}$ bond, resulting in a hydrogen in a bond-center (BC) site next to the $\mathrm{Si}-\mathrm{H}$ bond. The $\mathrm{H}-\mathrm{H}$ exchange then proceeds via an intermediate, metastable state, in which both $\mathrm{H}$ atoms are equally bonded to the $\mathrm{Si}$ atom, a configuration which we label $\equiv \mathrm{SiH}_{2}$. This configuration is discussed in more detail in Section V-D. In the $\equiv \mathrm{SiH}_{2}$ configuration the two $\mathrm{H}$ atoms can easily rotate; the $\mathrm{H}$ atom that was originally deeply bound can then jump to a BC position and diffuse away, completing the exchange.

Fig. 1 displays the exchange process schematically. Note that Fig. 1 includes neither all the atoms of the supercell nor all the atoms relaxed in our simulations. We use the following notation: hydrogen at a bond-center site is labeled $\mathrm{H}-\mathrm{BC}$; for the isolated -dangling bond we use DB, and if it is passivated by hydrogen we use $\mathrm{H}-\mathrm{DB}$ or $\mathrm{Si}-\mathrm{H}$, interchangeably; for hydrogen in a $\mathrm{BC}$ site next to a DB site, we use (H-BC,H-DB); finally, if $\mathrm{H}-\mathrm{BC}$ is far from a DB site we use $(\mathrm{H}-\mathrm{BC})+(\mathrm{H}-\mathrm{DB})$.

Fig. 1(a) is a schematic of the fully relaxed (H-BC,H-DB) complex which is the starting point for the exchange. The energy of the (H-BC,H-DB) complex is $0.15 \mathrm{eV}$ higher than the energy of $(\mathrm{H}-\mathrm{BC})+(\mathrm{H}-\mathrm{DB})$. This modest increase in energy does not constitute much of a barrier for an interstitial $\mathrm{H}$ atom to approach the $\mathrm{Si}-\mathrm{H}$ bond. Fig. 1(b) depicts the atomic positions in the intermediate $\equiv \mathrm{SiH}_{2}$ configuration; this configuration is discussed in more detail in Section V-D. Considering the full exchange process, we find that the energy barriers along Paths I and II in Fig. 1 are both smaller than $0.1 \mathrm{eV}$. Since the migration barrier for interstitial $\mathrm{H}$ is about $0.5 \mathrm{eV}$ [21], the barriers along paths I and II can easily be overcome at the modest temperatures at which interstitial $\mathrm{H}$ is mobile. The activation energy of the exchange process is therefore dominated by the energy cost of $0.15 \mathrm{eV}$ needed to place the interstitial (transport-level) hydrogen in a (H-BC,H-DB) state. The results of these calculations agree with the detailed experimental studies of Branz et al. [51], [52], as discussed thoroughly in [54]. 


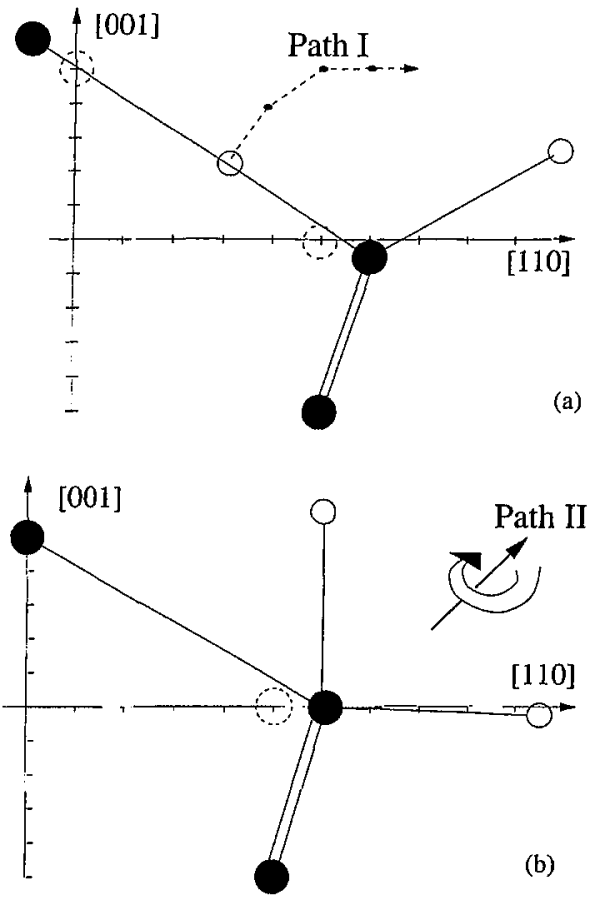

Fig. 1. Schematic illustration of the hydrogen-hydrogen exchange process. A dangling bond at atom $\mathrm{Si} 2$ passivated by a $\mathrm{H}$ atom (H-DB). The small open circle represents a hydrogen atom; the large filled circles represent silicon atoms. The solid lines represent bonds in the plane of the page [the (110) plane]; the double lines indicate bonds to the $\mathrm{Si} 3$ and $\mathrm{Si} 4$ atoms, which lie in front of, resp. behind, the plane of the page. (a) The bond-centered $\mathrm{H}$ atom moves by the path labeled I toward the dangling-bond region, resulting in a metastable $\equiv \mathrm{SiH}_{2}$ complex. The dotted circles represent the initial position of the silicon atom. The solid circles show the position of the $\mathrm{Si}$ atoms in the (H-BC, H-DB) complex. (b) In the $\equiv \mathrm{SiH}_{2}$ complex the two $\mathrm{H}$ atoms can "rotate" around the [111] direction, as schematically illustrated by Path II. To complete the exchange, the originally deeply bound $\mathrm{H}$ atom moves to a new $\mathrm{BC}$ position, along a path that is the equivalent of Path 1 .

\section{The $\equiv \mathrm{SiH}_{2}$ Complex in Bulk a-Si: $\mathrm{H}$ and at the $\mathrm{Si}_{-} \mathrm{SiO}_{2}$ Interface}

The $\equiv \mathrm{SiH}_{2}$ in the $c$-Si model discussed above is a locally stable configuration, but the barrier to go to the (H-BC,H-DB) configuration is less than $0.1 \mathrm{eV}$ high; the $\equiv \mathrm{SiH}_{2}$ would therefore not be stable for a long time. It is conceivable that certain sites in $a-\mathrm{Si}$ or at the $\mathrm{Si}-\mathrm{SiO}_{2}$ interface would provide a slightly greater stability of this complex, due to increased flexibility of the surrounding network.

One might speculate that the $\equiv \mathrm{SiH}_{2}$ complex will be a precursor for generation of $\mathrm{H}_{2}$, leaving a dangling bond behind. In fact, our calculations indicate that such a configuration, in which the $\mathrm{H}_{2}$ is still close to the dangling bond, is close in energy to the $\equiv \mathrm{SiH}_{2}$ complex (within $0.1 \mathrm{eV}$ ). Our preliminary investigations suggest that the barrier between these two configurations is about $1.5 \mathrm{eV}$ [55]. Conversely, adsorption of an $\mathrm{H}_{2}$ molecule may occur by the reverse of this $\mathrm{H}_{2}$ desorption process. The passivation of silicon dangling bonds by $\mathrm{H}_{2}$ at the $\mathrm{Si}(111)-\mathrm{SiO}_{2}$ interface has been experimentally observed to occur with activation energies of 1.66 or $1.51 \mathrm{eV}$ [8], [56], [57]. Since the dissociation energy for interstitial $\mathrm{H}_{2}$ in $\mathrm{SiO}_{2}$ is more than $4 \mathrm{eV}$, a process other than simple dissociation must be involved. Our findings indicate that the dissociation barrier is significantly reduced in the presence of a silicon dangling bond. Previously, it was assumed that $\mathrm{H}_{2}$ would dissociate into an interfacial $\mathrm{Si}-\mathrm{H}$ bond plus a hydrogen atom residing in $\mathrm{SiO}_{2}$. Based on our results we suggest that it is also possible for $\mathrm{H}_{2}$ to dissociate via the $\equiv \mathrm{SiH}_{2}$ complex forming an interfacial $\mathrm{Si}-\mathrm{H}$ bond and an $\mathrm{H}$ atom residing on the silicon side of the interface.

Finally, we suggest that the $\equiv \mathrm{SiH}_{2}$ complex might also play a role in defect formation in $a-\mathrm{Si}: \mathrm{H}$ and at the $\mathrm{Si}-\mathrm{SiO}_{2}$ interface. Since the complex is electrically active, light or free carriers could enhance the dissociation of the complex which may lead to formation of a dangling bond and an $\mathrm{H}_{2}$ molecule. This $\mathrm{H}_{2}$ molecule may diffuse away, leaving behind a dangling bond, i.e., an electrically active defect.

\section{SUMMARY AND OUTLOOK}

We have discussed a number of areas in which first-principles calculations have recently provided new insights into microscopic mechanisms relevant for hydrogenated amorphous silicon and for hydrogen at the $\mathrm{Si}-\mathrm{SiO}_{2}$ interface. $\mathrm{H}_{2}$ molecules may play a more important role than previously thought, because they diffuse and dissociate more easily than had been assumed. We have also discussed dissociation of the $\mathrm{Si}-\mathrm{H}$ bonds and our explanation for the enhanced stability of Si-D. With relevance for hydrogen diffusion, we have determined a low-energy exchange mechanism between interstitial and deeply bound hydrogen, which requires an activation energy of only $0.15 \mathrm{eV}$. The microscopic mechanism involves an intermediate $\equiv \mathrm{SiH}_{2}$ complex which may be involved in defect passivation and creation in both amorphous silicon and at the $\mathrm{Si}-\mathrm{SiO}_{2}$ interface.

The examples discussed in this paper were intended to convince the reader that first-principles calculations are a powerful tool for solving critical semiconductor problems. The first-principles approach will play an increasingly important role in device research, both as an effective aid in interpreting experimental results, and as a reliable predictor of new phenomena. Even within the realm of hydrogen interactions with silicon devices, important work still needs to be performed. For instance, further studies are definitely needed on hydrogen in $\mathrm{SiO}_{2}$. The complexity of the problem is greatly enhanced here by the amorphous nature of the host; the ability to perform first-principles calculations on increasingly larger systems (for instance, by the use of linear scaling methods) will play an important role here. The impact of first-principles calculations will also increase as they are integrated into multi-scale modeling approaches that are designed to address physical properties on a wide range of length and time scales.

Looking beyond current device structures, first-principles calculations based on density-functional theory can also be successfully applied to newer materials systems that must be engineered in future generations. These include SiGe, alternative dielectrics, etc. First-principles theory can certainly address the behavior of hydrogen in such structures-but also a host of other properties of these new classes of materials. This ability to accurately and reliably predict materials properties should be exceedingly valuable for exploration and implementation of new device structures. 


\section{ACKNOWLEDGMENT}

The authors gratefully acknowledge stimulating interactions and collaborations with J. Adams, C. Herring, K. Hess, W. Jackson, N. Johnson, N. Nickel, J. Neugebauer, S. Pantelides, R. Street, and N. Troullier., Some calculations were performed on the SGI-ORIGIN2000 machines at the National Center for Supercomputing Applications, Urbana, IL.

\section{REFERENCES}

[1] J. I. Pankove and N. M. Johnson, Eds., Hydrogen in Semiconductors. ser. Semiconductors and Semimetals. New York: Academic, 1991, vol. 34.

[2] R. A. Street, Hydrogenated Amorphous Silicon. Cambridge, U.K.: Cambridge Univ. Press, 1991.

[3] D. L. Staebler and C. R. Wronski, "Reversible conductivity changes in discharge-produced amorphous Si," Appl. Phys. Lett., vol. 31, p. 292, 1977.

[4] R. Helms and E. H. Poindexter, "The silicon silicon-dioxide system its microstructure and imperfections," Rep. Prog. Phys., vol. 57, p. 791, 1994.

[5] J. W. Lyding et al., "Nanoscale patterning and oxidation of $H$-passivated $\mathrm{Si}(100)-2 \times 1$ surfaces with an ultrahigh-vacuum scanning tunneling microscope," Appl. Phys. Lett., vol. 64, p. 2010, 1994.

[6] P. Avouris et al., "Breaking individual chemical bonds via STM-induced excitations," Surf. Sci., vol. 363, p. 368, 1996.

[7] K. L. Brower, "Dissociation kinetics of hydrogen-passivated (111) $\mathrm{Si}-\mathrm{SiO} 2$ interface defects," Phys. Rev. B, vol. 42, p. 3444, 1990.

[8] - "Chemical kinetics of hydrogen and (111) $\mathrm{Si}_{-} \mathrm{SiO}_{2}$ interface defects," Appl. Phys. Lett., vol. 57, p. 162, 1990.

[9] J. W. Lyding, K. Hess, and I. C. Kizilyalli, "Reduction of hot electron degradation in metal oxide semiconductor transistors by deuterium processing," Appl. Phys. Lett., vol. 68, p. 2526, 1996.

[10] B. R. Tuttle, W. McMahon, and K. Hess, "Hydrogen and hot electron defect creation at the $\mathrm{Si}(100) / \mathrm{SiO} 2$ interface of metal-oxide-semiconductor field effect transistors," Superlattices Microstruct., vol. 27, p. 229, 2000.

[11] R. Darwich, "Observation by infrared transmission spectroscopy and infrared ellipsometry of a new hydrogen-bond during light-soaking of a-Si-H," Philos. Mag., vol. 72, p. 363, 1995.

[12] W. Kohn and L.J. Sham, "Self-consistent equations including exchange and correlation," Phys. Rev. B, vol. 140, p. A1133, 1965.

[13] D. R. Hamann, M. Schlüter, and C. Chiang, "Norm-conserving pseudopotentials." Phys. Rev. Lett., vol. 43, p. 1494, 1979.

[14] N. Troullier and J. L. Martins, "Efficient pseudopotentials for plane-wave calculations," Phys. Rev B, vol. 43, p. 1993, 1991.

[15] C. G. Van de Walle, P. J. H. Denteneer, Y. Bar-Yam, and S. T. Pantelides, "Theory of hydrogen diffusion and reactions in crystalline silicon," Phys. Rev. B, vol. 39, p. $10791,1989$.

[16] C. G. Van de Walle, "Energies of various configurations of hydrogen in silicon," Phys. Rev. B, vol. 49, p. 4579, 1994.

[17] B. Tuttle and J. Adams, "Energetics of hydrogen in amorphous silicon: An ab initio study," Phys. Rev. B, vol. 57, p. $12859,1998$.

[18] N. M. Johnson, C. Herring, and C. G. Van de Walle, "Inverted order of acceptor and donor levels of monatomic hydrogen in silicon," Phys. Rev. Lett., vol. 73, p. 130, 1994.

[19] J. Neugebauer and C. G. Van de Walle, "Hydrogen in GaN - novel aspects of a common impurity," Phys. Rev. Lett., vol. 75, p. 4452, 1995.

[20] C. Herring and N. M. Johnson, Hydrogen in Semiconductors, J. I. Pankove and N. M. Johnson, Eds. New York: Academic, 1991, vol. 34, p. 279.

[21] A. Van Wieringen and N. Warmoltz, "Solubility and diffusivity of hydrogen in silicon," Physica, vol. 22, p. 849, 1956.

[22] P. E. Blöchl and E. Smargiassi et al., "First-principles calculations of self-diffusion constants in silicon," Phys. Rev. Lett., vol. 70, p. 2435, 1993.

[23] S. T. Pantelides, "Defects in amorphous-silicon - a new perspective," Phys. Rev. Lett., vol. 57, p. 2979, 1986.

[24] C. G. Van de Walle and J. Neugebauer, "Hydrogen interactions with self-interstitials in silicon," Phys. Rev. B, vol. 52, p. 14320, 1995.

[25] C. G. Van de Walle and R. A. Street, "Structure, energetics, and dissociation of Si-H bonds at dangling bonds in silicon," Phys. Rev. B, vol. 49, p. $14766,1994$.

[26] S. K. Estreicher, "Hydrogen-related defects in crystalline semiconductors-a theorist's perspective," Mat. Sci. Engr. Rep., vol. 14, p. 319, 1995.
[27] M. Bruel, "Silicon-on-insulator material technology," Electron. Lett., vol. 31, p. 1201, 1995.

[28] R. E. Norberg, D. J. Leopold, and P. A. Fedders, "Non-bonded hydrogen in a-Si:H," J. Non-Cryst. Solids, vol. 227-230, p. 124, 1998.

[29] P. A. C. G and P. A. Van de Walle, "Energetics and vibrational frequencies of interstitial $\mathrm{H} 2$ molecules in semiconductors," Phys. Rev. Lett., vol. 80, p. $2177,1998$.

[30] B. Tuttle, "Energetics and diffusion of hydrogen in SiO2," Phys. Rev. B, vol. 61, p. $4417,2000$.

[31] A. W. R. Leitch, V. Alex, and J. Weber, "Raman spectroscopy of hydrogen molecules in crystalline silicon," Phys. Rev. Lett., vol. 81, p. 421, 1998.

[32] R. E. Pritchard et al., "Interactions of hydrogen molecules with bondcentered interstitial oxygen and another defect center in silicon," Phys. Rev. B, vol. 56, p. 13118, 1997.

[33] Y. J. Chabal and C. K. N. Patel, "Solid hydrogen in amorphous-silicon phase-transition," Phys. Rev. Lett., vol. 53, p. 1771, 1984.

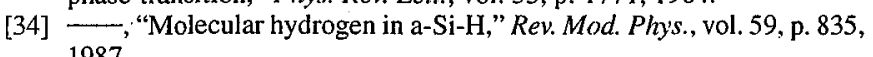

[35] A. Yokozawa and Y. Miyamoto, "First-principles calculations for charged states of hydrogen atoms in $\mathrm{SiO}_{2}$," Phys. Rev. $B$, vol. 55, p. 13783, 1997.

[36] P. E. Blöchl and J. H. Stathis, "Hydrogen electrochemistry and stressinduced leakage current in silica," Phys. Rev. Lett., vol. 83, p. 372, 1999

[37] B. Tuttle, "Hydrogen and P_b defects at the (111)Si-SiO2 interface: An ab initio cluster study," Phys. Rev. B, vol. 60, p. 2631, 1999.

[38] B. Tuttle and C. G. Van de Walle, "Structure, energetics, and vibrational properties of $\mathrm{Si}-\mathrm{H}$ bond dissociation in silicon," Phys. Rev. B, vol. 59, p. $12884,1999$.

[39] R. A. Street, C. C. Tsai, J. Kakalios, and W. B. Jackson, "Hydrogen diffusion in amorphous silicon," Philos. Mag. B, vol. 56, p. 305, 1987.

[40] S. Zafar and E. A. Schiff, "Hydrogen and defects in amorphous silicon," Phys. Rev. Lett., vol. 66, p. 1493, 1991.

[41] J.-H. Wei, M.-S. Sun, and S.-C. Lee, "A possible mechanism for improved light-induced degradation in deuterated amorphous-silicon alloy," Appl. Phys. Lett., vol. 71, p. 1498, 1997.

[42] S. Sugiyama, J. Yang, and S. Guha, "Improved stability against light exposure in amorphous deuterated silicon alloy solar cell," Appl. Phys. Lett., vol. 70, p. 378, 1997.

[43] T. Matsumoto, Y. Masumoto, and N. Koshida, "Reduction of luminescence degradation using deuterium-terminated porous silicon," Proc. Materials Research Soc. Symp. , vol. 452, p. 449, 1997.

[44] C. G. Van de Walle and W. B. Jackson, "Reduction of hot electron degradation in metal oxide semiconductor transistors by deuterium processing," Appl. Phys. Lett., vol. 69, p. 2441, 1996.

[45] T.-C. Shen et al., "Atomic-scale desorption through electronic and vibrational-excitation mechanisms," Science, vol. 268, p. 1590, 1995.

[46] P. Guyot-Sionnest, P. Dumas, Y. J. Chabal, and G. S. Higashi, "Lifetime of an adsorbate-substrate vibration: $\mathrm{H}$ on Si(111)," Phys. Rev. Lett., vol. 64, p. 2156, 1990.

[47] P. Guyot-Sionnest, P. H. Lin, and E. M. Miller, "Vibrational dynamics of the Si-H stretching modes of the $\mathrm{Si}(100) / \mathrm{H} 2 \times 1$ surface," $J$. Chem. Phys., vol. 102, p. 4269, 1995.

[48] O. Madelung, Ed., Data in Science and Technology: Semiconductors. Berlin, Germany: Springer-Verlag, 1991.

[49] R. Biswas, Y.-P. Li, and B. C. Pan, "Enhanced stability of deuterium in silicon," Appl. Phys. Lett., vol. 72, p. 3500, 1998.

[50] C. G. Van de Walle and R. A. Street, "Silicon-hydrogen bonding and hydrogen diffusion in amorphous-silicon," Phys. Rev. B, vol. 51, p. 10615, 1995.

[51] H. M. Branz, S. E. Asher, B. P. Nelson, and M. Kemp, "Hydrogen diffusion mechanism in amorphous silicon from deuterium tracer studies," J. Non-Cryst. Solids, vol. 164-166, p. 269, 1993.

[52] M. Kemp and H. M. Branz, "Hydrogen diffusion in a-Si:H: Solution of the tracer equations including capture by exchange," Phys. Rev. B, vol. 52, p. $13946,1995$.

[53] R. A. Street, "Amorphous silicon semiconductors-Pure and hydrogenated," in MRS Symposia Proc., vol. 95, A. Madan, M. Thompson, D. Adler, and Y. Hamakawa, Eds. Pittsburgh, PA, 1987, p. 13.

[54] B. Tuttle and C. G. Van de Walle, "Exchange of deeply trapped and interstitial hydrogen in silicon," Phys. Rev. B, vol. 59, p. 5493, 1999.

[55] B. Tuttle, private communication.

[56] E. Cartier, J. H. Stathis, and D. A. Buchanan, "Passivation and depassivation of silicon dangling bonds at the $\mathrm{Si} / \mathrm{SiO}_{2}$ interface by atomic-hydrogen," Appl. Phys. Lett., vol. 63, p. 1510, 1993.

[57] J. H. Stathis and E. Cartier, "Atomic hydrogen reactions with P-_b centers at the (100) Si/SiO2 interface," Phys. Rev. Lett., vol. 72, p. 2745, 1994. 


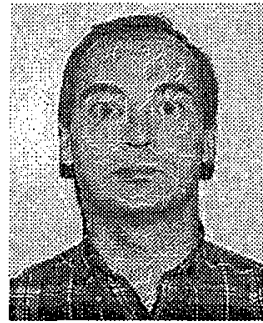

Chris G. Van de Walle (M'85) received the $\mathrm{Ph} . \mathrm{D}$. degree in electrical engineering in 1986 from Stanford University, Stanford, CA.

He was a Post-doctoral Scientist at the IBM T. J. Watson Research Center, Yorktown Heights, NY (1986-1988), a Senior Member of Research Staff at Philips Laboratories, Briarcliff Manor, NY (1988-1991), and an Adjunct Professor of Materials Science at Columbia University (1991). He joined the Xerox Palo Alto Research Center, Palo Alto, CA, in 1991. He develops and employs first-principles techniques to model the structure and behavior of semiconductors. He has performed extensive studies of semiconductor interfaces (including the development of a widely used model for band offsets) and of defects and impurities in semiconductors, with particular emphasis on doping problems and on the role of hydrogen. Recently, he has been focusing his attention on wide-band -gap semiconductors. He has published over 140 research papers and has given more than 40 invited talks at international conferences and numerous invited seminars. He has two patents.

Dr. Van de Walle is a Fellow of the American Physical Society and the recipient of a Humboldt Award for Senior U.S. Scientist. He chaired the Gordon Research Conference on Point and Line Defects in Semiconductors in 1998, the 23rd Conference on Physics and Chemistry of Semiconductor Interfaces in 1996, and the 7th Trieste Semiconductor Symposium on Wide-Band-Gap Semiconductors in 1992.

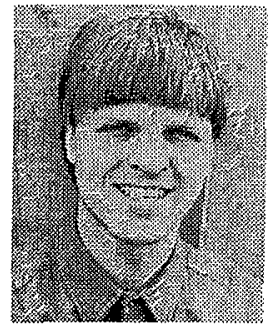

Blair R. Tuttle was born on June 9, 1969, in Syracuse, NY. He received the B.A. degree in May 1991 from Bates College, Lewiston, ME, and the Ph.D. degree in physics from the University of Illinois, Urbana-Champaign, in 1997.

Currently, he is a member of Computational Electronics Group, Beckman Institute, University of Illinois. His research involves atomic simulation of silicon-based materials. He primarily uses density functional calculations to study the microscopic aspects of various phenomena including hydrogen diffusion and defect passivation, tunneling currents in thin oxide, and electronic properties of organic nanostructures on silicon surfaces. 